\title{
The NEutrino Mediterranean Observatory (NEMO) Project
}

\section{Maurizio Spurio* for the NEMO Collaboration}

University of Bologna and INFN-Sezione di Bologna, Italy

E-mail: spurio@bo.infn.it

Since 1998 the NEMO Collaboration started a long term activity dedicated to improve deep-sea technologies and to characterize an optimal site for the installation of a $\mathrm{km} 3$ neutrino Telescope in the Mediterranean sea. More than 25 sea campaigns were performed to deploy and recover instrumented lines of multi-functional detectors, dedicated to survey the environmental characteristics and water optical properties at different sites. A site close to Capo Passero, $80 \mathrm{~km}$ from the southern coast of Sicily, Italy, at $3500 \mathrm{~m}$ below the Sea level shows excellent peculiarity to host a $\mathrm{km}^{3}$ underwater detector. A complete feasibility study, which considered all the detector critical components and the deployment procedures, has been carried out demonstrating that technological solutions exist for the realization of an underwater $\mathrm{km}^{3}$ detector. In parallel, an intense R\&D activity finalized at the development of new solutions for the sensors and the DAQ electronics is in progress. The realization of a technological demonstrator of the apparatus (the NEMO Phase 1 project) near the Catania harbour, at a depth of $2000 \mathrm{~m}$, is scheduled in 2006. The realization of a second demonstrator (a full tower with 16 storeys, 64 optical modules) at the Capo Passero site is foreseen for 2007.

International Europhysics Conference on High Energy Physics

July 21st - 27th 2005

Lisboa, Portugal

* Speaker. 


\section{Introduction}

High-energy neutrino observations can provide information on the most energetic phenomena in the Universe. Neutrinos may easily escape even from thick sources, are hardly absorbed during their propagation and are not deflected by magnetic fields. They are therefore the ideal candidate particles to point back to the sources of high-energy cosmic rays. Detectable sources of highenergy neutrinos are expected to exist in our Galaxy as well as at extra-Galactic distances [1]. This requires a detector with a huge sensitive volume, which should be effectively shielded from the overwhelming background from charged cosmic rays. The only viable solution nowadays is to build a large array of light sensors (photomultipliers, PMTs) in a transparent medium, such as water or ice. PMTs can then detect the Cherenkov light emitted by the charged leptons produced in charged-current interactions of neutrinos inside the apparatus or in its immediate surroundings.

An apparatus with an effective surface of the order of one $\mathrm{km}^{2}$ (or a sensitive volume of $\sim 1 \mathrm{~km}^{3}$ ) is needed for neutrino astrophysics. So far, smaller scale detectors have been implemented or are under construction [2], demonstrating the feasibility of the technique of the Cherenkov light detection. However, the construction of an underwater $\mathrm{km}^{3}$ detector needs further improvements of the technologies by means of appropriate R\&D studies, since probably some of the technical solutions used for smaller scale detectors are not scalable to such a large detector.

The NEMO (NEutrino Mediterranean Observatory) Collaboration was set up in 1998 with the aim to carry out the necessary $R \& D$ towards a $\mathrm{km}^{3}$ neutrino telescope in the Mediterranean Sea. The Mediterranean Sea seems the natural choice for the installation of a detector in the Northern hemisphere complementary to ICECUBE [3]. The activity has been mainly focused on the search and characterization of an optimal site for the installation, on the development and test of key technologies for the $\mathrm{km}^{3}$ underwater detector, on the realization of small scale technological demonstrators, and on the definition of a general architecture of the detector.

\section{Site selection and characterization}

The installation of the $\mathrm{km}^{3}$ detector needs a complete knowledge of the site physical and oceanographic characteristics over a long time period. The Mediterranean Sea offers optimal conditions to locate the Telescope. A candidate site should be deep enough, to minimize the background from secondary cosmic rays; far from shelf breaks and submarine canyons, for detector stability. The water should offer optimal optical transparency, to maximize the detector performances in terms of efficiency and angular resolution. The site should have stable deep sea currents, to reduce stresses on mechanical structures and to reduce the stimulation of bioluminescent organisms. A low level of sedimentation should be present. Finally, it should be located not too far from the coast and to existing infrastructures, for easy access for sea operations, and to reduce the costs for installation and maintenance.

After 25 sea campaigns since 1998, a site located close to Capo Passero, in the South-East part of Sicily in the Ionian Sea, results as the best candidate. It consists of a wide abyssal plateau at a depth of about $3500 \mathrm{~m}$, at less than $80 \mathrm{~km}$ from the shore, and far from shelf breaks or submarine canyons. Capo Passero offers excellent naval facilities (harbours of Catania, Siracusa and Augusta) and the neighbouring infrastructures of the Laboratori Nazionali del Sud of INFN. 
For the Capo Passero site, the nature and structure of the seabed were studied in details, as well as the optical properties of the water. Water transparency to electromagnetic radiation can be characterized by means of quantitative parameters: the absorption length $L_{a}$ and the scattering length $L_{b}$. Scattering refers to processes in which the direction of the photon is changed without any other alteration. In pure water, light absorption and scattering are strongly wavelength dependent [4]. In particular the light transmission length $L_{c}(\lambda)$ (combined effect of absorption and scattering: $\left.1 / L_{c}=1 / L_{a}+1 / L_{b}\right)$ in pure water is extremely favoured in the range $350 \div 550 \mathrm{~nm}$, overlapping the region in which PMTs usually reach the highest quantum efficiency. The effective volume of a Neutrino Telescope depends on the light absorption, while the track reconstruction capabilities on the light scattering. The in situ measurements show [5] that the absorption length is $L_{a}=$ $67 \pm 13.5 \mathrm{~m}$ in the blue region $(440 \mathrm{~nm})$ and it does not depends from the depth (for depths larger than $2500 \mathrm{~m}$ ). The corresponding value of the attenuation length is $L_{c}=35.6 \pm 2.5 \mathrm{~m}$.

The PMTs counting rate in an undersea detector is strongly affected by the decay of radioactive elements in water (mainly ${ }^{40} K$ ) and the luminescence produced by biological entities. The measured baseline of optical noise rate (using a $8^{\prime \prime}$ PMT with a threshold of 0.3 p.e.) amounts to $28 \mathrm{kHz}$ at a depth of $3000 \mathrm{~m}$. Beyond a depth of about $2500 \mathrm{~m}$, the biological source of optical noise dramatically disappears, and the biological signal limits itself to rare high rate spikes: the measured burst rate time fraction on each PMT is $\sim 0.2 \%$ (see [2],pag. 93). The water current intensity and direction were measured over more than 7 years of data taking; the currents at a depth of about $3000 \mathrm{~m}$ appear to be low and regular $(2-3 \mathrm{~cm} / \mathrm{s}$ average; $12 \mathrm{~cm} / \mathrm{s}$ maximum). A sediment trap, installed at about $110 \mathrm{~m}$ above the seabed, which collected sediment samples over periods of 15 days, measured a rather constant low particle flux (average $20 \mathrm{mg} \mathrm{m}^{-2} \mathrm{~d}^{-1}$ ).

\section{Preliminary project for ${\mathbf{a ~} \mathbf{~}^{3}}^{3}$ detector}

Preliminary Monte Carlo simulations (see [2],pag. 104) show that one can reach an effective area of $\sim 1 \mathrm{~km}^{2}$ for muon energies larger than few TeV, by filling a water volume of $\sim 1 \mathrm{~km}^{3}$ with few thousand of optical sensors. Taking into account some mechanical constraints, our studies favour a detector architecture composed by a square array of structures, called towers (see [2], pag. 5). The response of different detector configurations to muons with different energies was studied using the software package developed by the ANTARES collaboration (see [2],pag. 109). As an example (see [2], pag. 114), a detector layout made by a $9 \times 9$ array of 81 NEMO towers, with 5832 PMT has an effective area of $1 \mathrm{~km}^{2}$ at $E_{\mu} \geq 10 \mathrm{TeV}$. This effective area is obtained using quality cuts on the track reconstruction, which allow a good angular resolution: the median angle between true and reconstructed muon tracks was better than $0.2^{\circ}$. In the preliminary design, each tower is composed by a sequence of 18 storeys (that host the instrumentation); the storey is made by a 15 $\mathrm{m}$ long structure, hosting two optical modules (one down looking and one looking horizontally) at each end. The spacing between storeys will be $40 \mathrm{~m}$, with each storey rotated around the vertical axis by $90^{\circ}$ with respect to the up and down adjacent ones.

\section{Technological demonstrators}

A prototyping activity has been launched in order to implement reduced-scale demonstrators 
of the apparatus and to validate all the technical solutions proposed. The NEMO Phase 1 project is under realization at the underwater test site of the INFN Laboratori Nazionali del Sud, located at $2000 \mathrm{~m}$ depth and at a distance of about $25 \mathrm{~km}$ from the Catania harbour. In January 2005, submarine termination panels were connected on an electro-optical cable so that a Remotely Operated Vehicle (ROV) was used to connect underwater instrumentation: an on-line seismic observatory developed by INGV (the Italian National Institute for Geophysics and Volcanology) and a prototype station for acoustic background measurements. The acoustic station has been taking data continuously since then [6]. The NEMO Phase 1 apparatus will consist of a 4 storey tower, hosting the optical modules (OMs) and the instrumentation, interlinked by a system of cables and anchored on the seabed. Each storey (15 m long), will host two OMs at each end. Such modules consist of pressure-resistant glass spheres containing a large $\left(10^{\prime \prime}\right)$ hemispheric photomultiplier and its front-end electronics. Such a structure is kept vertical by appropriate buoyancy on the top. An innovative design has been exploited for the junction box, which will be built as two containers inserted one into the other: a pressure-resistant steel vessel inserted inside a corrosion-resistant fibreglass container filled with mineral oil. The data communication system exploits a standard, synchronous telecommunication protocol (SDH) (see [2], pag. 160). A Dense Wavelength Division Multiplexing (DWDM) system has been designed to allow high-bandwidth point-to-point bidirectional communications between the shore and each single storey of the towers, with a reasonable number of optical fibres. The towers will also feature: a redundant positioning system, which includes compasses, inclinometers and acoustic triangulation devices; a timing calibration system, and several environmental sensors. This sub-project will be completed during 2006.

During 2007, the NEMO Phase 2 will start. The objectives of this sub-project is the realization of an underwater infrastructure at a depth of $3500 \mathrm{~m}$ on the Capo Passero site. A building located inside the harbour area of Portopalo di Capo Passero has been already acquired. This building (which will be renovated starting from 2006) will host a shore station for the power feeding and the data acquisition systems. From this store station, a $100 \mathrm{~km}$ electro optical cable will connect the underwater infrastructures. The purchase of the electro-optical cable (with a transmission power of about $40 \mathrm{~kW}$ ) is at present under way. At the end of the electro-optical cable, a Junction box (similar to the one employed in Nemo Phase 1) will be deployed and a 16 storey tower (with 64 OMs) will be installed. The storeys and the OMs will be of the same type as the ones used for the NEMO Phase 1. This sub-project will allow to test the detector structure installation procedures at $3500 \mathrm{~m}$ depth, and to perform a long term monitoring of the site.

\section{References}

[1] J.G. Learned and K. Mannheim, Annu. Rev. Part. Sci. 2000 (50),679

[2] Proc. of the $1^{\text {st }}$ VLVnT Workshop, Amsterdam (2003) (http://www.vlvnt.nl/proceedings.pdf/)

[3] J. Ahrens et al., Astrop. Phys. 20(2004) 507.

[4] J.A. Aguilar et al., Astrop. Phys. 23(2005) 131.

[5] A. Capone et al., Nucl. Instr. Meth. A487 (2002),423.

[6] G. Riccobene (NEMO Collaboration) XXIX ICRC, Pune 3-10 Aug, HE24. 